\title{
SEMI-GROUPS OF OPERATORS
}

\author{
R. S. PHILLIPS ${ }^{1}$
}

1. Introduction. I should like to present some of the recent developments in the theory of one-parameter semi-groups of linear bounded operators. Actually this field is relatively new, having its origin in the work of M. H. Stone [16] on one-parameter groups of unitary operators in Hilbert space which appeared in 1930. By now the subject has an extensive literature, the definitive work being E. Hille's [4] Functional analysis and semi-groups, published in 1948. An adequate bibliography for the material in this field published prior to 1948 can be found in Hille's treatise. I shall limit my remarks to developments since 1948, emphasizing perhaps unduly my own contributions. Further I shall not discuss in any detail the applications to partial differential equations of parabolic type, although this has been one of the most gratifying aspects of the theory; this material can be found in papers by W. Feller [1], E. Hille [7], and K. Yosida $[20 ; 21]$.

Let $\mathfrak{S} \equiv[T(\xi) ; \xi>0]$ be a one-parameter family of linear bounded operators defined on a complex Banach space $\mathfrak{X}$ to itself and satisfying the product law

$$
T\left(\xi_{1}+\xi_{2}\right)=T\left(\xi_{1}\right) T\left(\xi_{2}\right), \quad \xi_{1}, \xi_{2}>0 .
$$

We shall further assume that $T(\xi) x$ is a continuous function of $\xi$ for $\xi>0$ and each $x \in \mathfrak{X}$, that is, we assume that $\mathbb{S}$ is continuous in the strong operator topology. The infinitesimal operator is defined as the limit in norm

$$
\lim _{\eta \rightarrow 0+} \frac{T(\eta)-I}{\eta} x=A_{0} x
$$

wherever this limit exists, the domain of $A_{0}$ (in symbols $\mathfrak{D}\left(A_{0}\right)$ ) being the set of elements for which the limit exists. It is easy to see that $A_{0}$ is a linear operator. However, $A_{0}$ need not be bounded nor even closed; its least closed extension, when it exists, will be called the infinitesimal generator of $\subseteq$ and will be designated by $A$. The latter operator plays a basic role in the theory. For $x \in \mathscr{D}\left(A_{0}\right)$ we have

An address delivered before the Summer Meeting of the Society in Laramie on September 2, 1954 by invitation of the Committee to Select Hour Speakers for Annual and Summer Meetings; received by the editors October 8, 1954.

${ }_{1}^{1}$ This manuscript was prepared while the author was holding a John Simon Guggenheim Fellowship. 


$$
\frac{d T(\xi) x}{d \xi}=A_{0} T(\xi) x=T(\xi) A_{0} x, \quad \xi>0 .
$$

Moreover, denoting the range space for $\subseteq \mathfrak{S}$ by

$$
\mathfrak{X}_{0} \equiv \underset{\xi>0}{\bigcup} T(\xi)[\mathfrak{X}]
$$

it can be shown that

$$
\left[\mathfrak{D}\left(A_{0}\right)\right]^{-}=\left[\mathfrak{X}_{0}\right]^{-} .
$$

In applications it is usually required that the semi-group satisfy an initial condition of the sort

$$
\lim _{\xi \rightarrow 0^{+}} T(\xi) x=x, \quad x \in \mathfrak{X} .
$$

Now $\lim _{\xi \rightarrow 0+} T(\xi) x=x$ for each $x \in \mathfrak{X}_{0}$ whereas this relation is never satisfied for $x \in\left[\mathfrak{X}_{0}\right]$-. This suggests that we restrict the operators $T(\xi)$ to the subspace $\mathfrak{Y} \equiv\left[\mathfrak{X}_{0}\right]-$, in which case we obtain a semi-group of operators on $\mathfrak{V}$ to itself. The range space for the restricted semigroup is again $\mathfrak{X}_{0}$ so that the above initial condition is now satisfied at least by a set of vectors dense in the space. Instead of considering the restricted operators, it is simpler to assume at the outset that $\mathfrak{X}_{0}$ is dense in $\mathfrak{X}$. If in addition $\|T(\xi)\|$ is bounded near the origin, then the condition $\left(\mathrm{C}_{0}\right)$ is satisfied in its entirety and we say that $\mathfrak{S}$ is of class $\left(\mathrm{C}_{0}\right)$. For such semi-groups $A_{0}$ is itself closed so that $A=A_{0}$.

Many problems in classical analysis are subsumed under the theory of semi-groups of linear bounded operators. This is in particular true of Cauchy's problem which arises in the theory of partial differential equations. Abstracting from this, E. Hille $[6 ; 7]$ has introduced an abstract Cauchy problem, a modification of which is

ACP. Given a closed linear operator $U$ with domain and range in $\mathfrak{X}$, and given an element $y_{0} \in \mathfrak{X}$, find a function $y(\xi) \equiv y\left(\xi ; y_{0}\right)$ on $[0, \infty)$ to $\mathfrak{X}$ such that

(1) $y(\xi)$ is continuously differentiable on $[0, \infty)$,

(2) $y(\xi) \in \mathfrak{D}(U)$ and $U[y(\xi)]=y^{\prime}(\xi)$ for each $\xi>0$,

(3) $\lim _{\xi \rightarrow 0+} y\left(\xi ; y_{0}\right)=y_{0}$.

As an example of the above consider the classical diffusion problem in the Banach space $C[-\infty, \infty]$ :

$$
\begin{array}{rlrl}
\partial u / \partial \xi & =\partial^{2} u / \partial x^{2}, & -\infty<x<\infty, & \xi>0 ; \\
\lim _{\xi \rightarrow 0+} u(\xi, x) & =y_{0}(x), & -\infty<x<\infty .
\end{array}
$$

Here one defines the closed linear operator $U$ by 


$$
\begin{aligned}
{[U y](x) } & =y^{\prime \prime}(x) \\
\mathfrak{D}(U) & =\left[y ; y(x), y^{\prime}(x)\right. \text { absolutely continuous }
\end{aligned}
$$

$$
\text { and } \left.y, y^{\prime \prime} \in C[-\infty, \infty]\right] \text {. }
$$

If $y(\xi)=y\left(\xi ; y_{0}\right)$ satisfies the ACP and has the realization $\phi(\xi, x)$ so that $y(\xi)=\phi(\xi, \cdot)$, then condition (1) means that $\phi(\xi, x)$ is continuously differentiable with respect to $\xi, \xi>0$, uniformly with respect to $x$; condition (2) implies that $\phi(\xi, \cdot) \in \mathfrak{D}(U)$ for each $\xi>0$ and that $u=\phi(\xi, x)$ satisfies the first relation in (1.5); and finally condition (3) requires that $u=\phi(\xi, x)$ satisfies the second relation in (1.5) uniformly with respect to $x .^{2}$

There is a close connection between the ACP and the theory of semi-groups. Indeed, if $\subseteq=[T(\xi)]$ is a semi-group of class $\left(\mathrm{C}_{0}\right)$ and $y_{0} \in \mathfrak{D}(A), A$ being the infinitesimal generator of $\mathfrak{S}$, then the relation (1.3) shows that $y\left(\xi ; y_{0}\right) \equiv T(\xi) y_{0}$ is continuously differentiable in $\xi$ for $\xi \geqq 0$ and that $A[y(\xi)]=y^{\prime}(\xi)$, whereas condition $\left(\mathrm{C}_{0}\right)$ implies that $\lim _{\xi \rightarrow 0+} y\left(\xi ; y_{0}\right)=y_{0}$. Thus $y\left(\xi ; y_{0}\right)=T(\xi) y_{0}$ satisfies the ACP for each $y_{0} \in \mathfrak{D}(A)$. Conversely we have (see R. S. Phillips [14])

THEOREM 1.1. Let $U$ be a closed linear operator with dense domain and a nonempty resolvent set. Suppose for each $y_{0} \in \mathfrak{D}(U)$ that there exists a unique solution $y\left(\xi ; y_{0}\right)$ to the ACP. Then $U$ is the infinitesimal generator of a semi-group $[T(\xi)]$ of class $\left(\mathrm{C}_{0}\right)$ such that $T(\xi) y_{0}=y\left(\xi ; y_{0}\right)$ for each $y_{0} \in \mathfrak{D}(U)$.

We remark that the semi-group property of the solution to the $\mathrm{ACP}$ is an easy consequence of the uniqueness assumption. However, the surprising feature in the above assertion is that $y\left(\xi ; y_{0}\right)$ is a continuous function of the initial data or, otherwise expressed, that the solution is stable relative to the initial data; this follows from the fact that the semi-group operators $T(\xi)$ are bounded.

2. Generation of semi-groups. In view of the above connection between the ACP and semi-groups of operators, the question of when a closed linear operator $U$ generates a semi-group is of particular interest. To begin with one can ask whether such a problem is well set in the sense of Hadamard, that is, one can ask whether

(1) there exists at most one solution,

2 If $\mathfrak{X}$ is a Lebesgue space rather than $C[-\infty, \infty]$, then the interpretation of conditions (1), (2), and (3) of ACP is quite different since the limits are then limits in the mean. However, it is possible to construct a realization $\phi(\xi, x)$ of $y\left(\xi ; y_{0}\right)$ such that $\phi(\xi, x)$ is absolutely continuous in $\xi, \xi \geqq 0$, for each $x, \partial \phi(\xi, x) / \partial \xi$ exists almost everywhere in the product space, and for almost all $\xi$ this function is a representation of $U[y(\xi)]$. 
(2) there exists at least one solution,

(3) the solution is stable, that is, the semi-group is a continuous function of the infinitesimal generator.

We shall take these conditions up in order. As for (1), it can be shown rather easily and under quite general conditions that a closed linear operator generates at most one semi-group. ${ }^{3}$ On the other hand the existence question is fairly deep and permits many kinds of answers. Indeed, Theorem 1.1 provides an answer of a sort for this problem. However, for many applications it is more convenient to have a criteria which depends more directly on the properties of $U$ itself. Condition (3) will be discussed in the next section.

The most satisfactory answer to (2) is given in terms of the resolvent of $U$, namely, $R(\lambda ; U)$. The motivation here is as follows. For a semi-group $\subseteq \equiv[T(\xi)]$ continuous in the strong operator topology, the

$$
\lim _{\xi \rightarrow \infty} \xi^{-1} \log \|T(\xi)\| \equiv \omega_{0}
$$

exists; the quantity $\omega_{0}$, called the type of $\mathfrak{S}$, is finite or $-\infty$, but never $+\infty$. Consequently if $\subseteq$ is of class $\left(\mathrm{C}_{0}\right)$, it is possible to take the Laplace transform of $T(\xi) x$. It turns out that

$$
R(\lambda ; A) x=\int_{0}^{\infty} e^{-\lambda \xi} T(\xi) x d \xi, \quad \Re(\lambda)>\omega_{0}
$$

here the right member is obtained as an abstract Bochner integral in $\mathfrak{X}$. Thus our problem is in essence one of determining when $R(\lambda ; U)$ is the Laplace transform of an operator-valued function. Phrased in this way, the problem has its classical analogue (see D. V. Widder [18, Chap. VII]), and the criteria which we shall obtain for $R(\lambda ; U)$ are the prototypes of the classical criteria. However, the proofs required in the present problem differ radically from the classical case in that they no longer depend on compactness arguments but rather on the properties of the resolvent function.

The first result in this direction, and for many purposes the most useful, was obtained independently by E. Hille $[4 ; 5]$ and $\mathrm{K}$. Yosida [19] in 1948. A complete characterization of the infinitesimal generator for semi-groups of class $\left(\mathrm{C}_{0}\right)$ was not found until 1953 when the following theorem was published almost simultaneously by $W$. Feller [3], I. Miyadera [1], and R. S. Phillips [12].

${ }^{3}$ More precisely, a closed linear operator can generate at most one semi-group of class (A) (see \$4). 
THEOREM 2.1. A necessary and sufficient condition that a closed linear operator $U$ generate a semi-group $[T(\xi)]$ of class $\left(\mathrm{C}_{0}\right)$ is that $\mathfrak{D}(U)$ be dense in $\mathfrak{X}$ and that there exist real constants $M$ and $\omega$ such that

$$
\|[R(\lambda ; U)] n\| \leqq M(\lambda-\omega)^{-n}
$$

for all $\lambda>\omega$ and $n=1,2,3, \cdots$. In this case $\|T(\xi)\| \leqq M \exp (\omega \xi)$.

We obtain the Hille-Yosida theorem as a corollary, viz.

CoRollary. If $U$ is a closed linear operator with dense domain, if $R(\lambda ; U)$ exists for $\lambda>0$, and if

$$
\lambda\|R(\lambda ; U)\| \leqq 1
$$

for all $\lambda>0$, then $U$ is the infinitesimal generator of a semi-group $[T(\xi)]$ of class $\left(\mathrm{C}_{0}\right)$ such that $\|T(\xi)\| \leqq 1$ for all $\xi>0$.

In all of the present applications of semi-group theory to diffusion theory, the above corollary has been used to establish the existence of a semi-group solution. In order to see what is involved here, we shall again consider the differential equation (1.5), the associated operator $U$ being given by (1.6). It is clear that $\mathfrak{D}(U)$ is dense in $C[-\infty, \infty]$. Further $R(\lambda ; U) f$ is obtained as the solution to

$$
(\lambda I-U) v \equiv \lambda v-v^{\prime \prime}=f
$$

with $v \in \mathfrak{D}(U)$ so that

$$
[R(\lambda ; U) f](x)=\left(2 \lambda^{1 / 2}\right)^{-1} \int_{-\infty}^{\infty} \exp \left(-\lambda^{1 / 2}|x-y|\right) f(y) d y
$$

$$
\lambda>0 .
$$

An elementary calculation shows that

$$
\begin{aligned}
\|R(\lambda ; U) f\| & \leqq \sup _{x}\left(2 \lambda^{1 / 2}\right)^{-1} \int_{-\infty}^{\infty} \exp \left(-\lambda^{1 / 2}|x-y|\right) d y \cdot\|f\| \\
& =\lambda^{-1}\|f\| .
\end{aligned}
$$

The conditions of the corollary are therefore satisfied and it follows that (1.5) has a semi-group solution of class $\left(\mathrm{C}_{0}\right)$.

The advantage obtained in using the criterion (2.4) rather than (2.3) clearly lies in the fact that the former requires a bound only for the first power of the resolvent operator, whereas the latter requires a bound on all of the positive integral powers of the resolvent. In order to make such conditions on $U$ less stringent we are led to consider more general classes of semi-groups. 
If we wish to keep the theory within the present framework, there is only one condition which can be relaxed and that is the behavior of the semi-group near the origin. Indeed, assuming strong measurability instead of strong continuity for $\xi>0$ does not in fact broaden the class of semi-groups since strong measurability can be shown to imply strong continuity (see R. S. Phillips [9]). The assumption of weak measurability, on the other hand, does not imply strong continuity and leads to a considerably more general theory as W. Feller [2] has shown. However, the added generality obtained by so weakening the hypotheses does not, in our opinion, compensate for the concomitant loss of detail in the theory.

A useful class of semi-groups can be defined as

Definition. A semi-group of linear bounded operators $[T(\xi)]$ is said to be of class (1, A) if it satisfies the conditions

(1) $T(\xi)$ is continuous in the strong operator topology for $\xi>0$,

(2) $\int_{0}^{1}\|T(\xi)\| d \xi<\infty$,

(3) $\lim _{\lambda \rightarrow \infty} \lambda \int_{0}^{\infty} e^{-\lambda \xi} T(\xi) x d \xi=x$ for each $x \in \mathfrak{X}$.

Condition (2) in this definition allows $\|T(\xi)\|$ to become unbounded as $\xi \rightarrow 0+$, whereas condition (3) replaces $\left(\mathrm{C}_{0}\right)$ by strong Abel summability to the identity at $\xi=0$. For semi-groups of class $(1, A)$ the infinitesimal operator $A_{0}$ need not be closed; however, the least closed extension of $A_{0}$, namely the infinitesimal generator $A$, again satisfies the relation (2.2) in the half-plane $\Re(\lambda)>\omega_{0}$. This shows, incidentally, that the resolvent set for $A$ contains a right half-plane. Moreover, it is easy to see that $\|R(\lambda ; A)\|$ is bounded in every halfplane $\Re(\lambda) \geqq \omega>\omega_{0}$. We now have (R. S. Phillips [13])

Theorem 2.2. A necessary and sufficient condition that a closed linear operator $U$ be the infinitesimal generator of a semi-group $[T(\xi)]$ of class $(1, \mathrm{~A})$ is that

(1) $\mathfrak{D}(U)$ is dense in $\mathfrak{X}$;

(2) $\|R(\lambda ; U)\|=O(1 / \lambda)$ as $\lambda \rightarrow \infty$;

(3) there exist real constants $M$ and $\omega$ such that

$$
\int_{0}^{\infty} \lambda^{n-1}\left\|R^{(n)}(\lambda+\omega ; U)\right\| d \lambda \leqq(n-1) ! M
$$

for $n=1,2,3, \cdots$.

Condition (3) can be replaced by

$\left(3^{\prime}\right)$ there exists a non-negative measurable function $\phi(\xi)$ and a constant $\omega$ such that $\int_{0}^{\infty} e^{-\omega \xi} \phi(\xi) d \xi<\infty$ and

$$
\left\|R^{(n)}(\lambda ; U)\right\| \leqq \int_{0}^{\infty} e^{-\lambda \xi \xi n} \phi(\xi) d \xi
$$


for all real $\lambda>\omega$ and integers $n \geqq 0$. If condition $\left(3^{\prime}\right)$ is verified, then $\|T(\xi)\| \leqq \phi(\xi)$ almost everywhere and if in addition $\phi(\xi)$ is bounded near the origin, then $[T(\xi)]$ is of class $\left(\mathrm{C}_{0}\right)$.

In the numerical case, condition (2.8) is a necessary and sufficient condition that $R(\lambda ; U)$ be a Laplace-Stieltjes transform. However, (2.8) together with the properties of the resolvent operator (and (1) and (2)) suffice to make $R(\lambda ; U)$ a Laplace transform.

The following corollary generalizes a theorem due to E. Hille [6].

Corollary. $A$ necessary and sufficient condition that a closed linear operator $U$ be the infinitesimal generator of a semi-group of class $(1, \mathrm{~A})$ is that

(1) $\mathfrak{D}(U)$ is dense in $\mathfrak{X}$;

(2) $\|R(\lambda ; U)\|=O(1 / \lambda)$ as $\lambda \rightarrow \infty$;

(3) there exists a family $[T(\xi) ; \xi>0]$ of linear bounded operators strongly continuous for $\xi>0$ with $\int_{0}^{\infty} e^{-\omega \xi}\|T(\xi)\| d \xi<\infty$ for some real $\omega$ such that

$$
R(\lambda ; U) x=\int_{0}^{\infty} e^{-\lambda \xi} T(\xi) x d \xi
$$

holds for all $\lambda>\omega$ and all $x \in \mathfrak{X}$.

Theorem 2.2 can be used to obtain the following sufficiency criterion: $A$ closed linear operator $U$ with dense domain generates a semigroup of class $(1, \mathrm{~A})$ if

$$
\|R(\sigma+i \tau ; U)\| \leqq M /\left(\sigma-\omega+|\tau|^{\beta}\right),
$$$$
\sigma>\omega
$$

providing $\beta>1 / 2$.

Condition (2.11) has the virtue of requiring a bound only on $\|R(\lambda ; U)\|$ and it has some advantage over the Hille-Yosida criterion in that $M$ is not restricted to the value one. The above criterion with $\beta=0$ is not sufficient that $U$ be the infinitesimal generator of a strongly continuous semi-group of linear bounded operators (see R. S. Phillips [13]). As we shall see in $\$ 4$, if $0<\beta \leqq 1 / 2$ then the operator $U$ does generate a semi-group of linear bounded operators; however, in this case the semi-group may belong to a more general class than $(1, \mathrm{~A})$.

3. Perturbation theory. Returning to the generation problem, we recall that stability was the third property needed in order that this problem be well set. We shall establish stability as a by-product of a perturbation theory for semi-groups of class $(1, A)$. In what follows we shall perturb the infinitesimal generator by adding to it certain 
linear operators (cf. R. S. Phillips $[12 ; 13]$ ). One would expect that those semi-group properties which persist under such perturbations are the more basic semi-group properties and that the significant theorems of the subject should evolve about these properties.

We shall consider only semi-groups of class $(1, \mathrm{~A})$. If $A$ is the infinitesimal generator of such a semi-group, we denote the corresponding semi-group by $[T(\xi ; A)]$. A linear operator $B$ will be said to belong to the perturbing class $\mathfrak{P}(A)$ if (i) $\mathfrak{D}(B)=\mathfrak{D}(A)$, (ii) $B R(\lambda ; A)$ is a bounded linear operator for some $\lambda$ in the resolvent set of $A$, and (iii) $\int_{0}^{1}\|B T(\xi ; A)\|_{A} d \xi<\infty$, where the subscript $A$ means that the norm is taken relative to the subspace $\mathfrak{D}(A)$. We remark that an operator $B \in \mathfrak{P}(A)$ may be unbounded and need not even have a closed extension. However, such a $B$ will always have a uniquely defined extension $\widetilde{B}$ such that $\widetilde{B} T(\xi ; A)$ is linear and bounded on $\mathfrak{X}$ for each $\xi>0$. If $B$ does possess a closed extension, then condition (ii) is automatically satisfied by the closed graph theorem.

In order to illustrate the above conditions we again consider the diffusion equation (1.5) whose solution is given by a semi-group $[T(\xi)]$ of class $\left(\mathrm{C}_{0}\right)$ with infinitesimal generator $U$ defined by (1.6). As is well known, the Weierstrass-Gauss kernel furnishes a representation for this semi-group, namely,

$$
[T(\xi) f](x)=\frac{1}{2}(\pi \xi)^{-1 / 2} \int_{-\infty}^{\infty} \exp \left[-(x-y)^{2} / 4 \xi\right] f(y) d y, \quad \xi>0 .
$$

We now define the linear operator $B$ with domain $\mathscr{D}(U)$ by

$$
[B f](x)=b(x) f^{\prime}(x), \quad f \in \mathfrak{D}(U),
$$

where $b \in C[-\infty, \infty]$. According to the relation (2.6)

$$
\begin{aligned}
& {[B R(\lambda ; U) f](x)} \\
& \quad=\frac{1}{2} b(x) \int_{-\infty}^{\infty} \operatorname{sgn}(y-x) \exp \left[-\lambda^{1 / 2}|x-y|\right] f(y) d y
\end{aligned}
$$

so that the operator $B R(\lambda ; U)$ is bounded for $\Re(\lambda)>0$. In the present case $\mathfrak{D}(U) \supset \mathfrak{X}_{0}$ and

$$
\begin{aligned}
& {[B T(\xi) f](x)} \\
& \quad=\frac{1}{4} b(x)\left(\pi \xi^{3}\right)^{-1 / 2} \int_{-\infty}^{\infty}(y-x) \exp \left[-(x-y)^{2} / 4 \xi\right] f(y) d y .
\end{aligned}
$$

It is easy to see from (3.4) that

$$
\|B T(\xi)\| \leqq\|b\|(\pi \xi)^{-1 / 2}, \quad \xi>0,
$$


so that $\int_{0}^{1}\|B T(\xi)\| d \xi<\infty$. It follows that $B \in \mathfrak{B}(U)$. Thus $B$ is an unbounded perturbing operator for the semi-group (3.1).

Our basic perturbation theorem is

THEOREM 3.1. Let $[T(\xi ; A)]$ be a semi-group of class $(1, \mathrm{~A})$ and suppose that $B \in \mathfrak{B}(A)$. Then $A_{1}=A+B$ with domain $\mathfrak{D}\left(A_{1}\right)=\mathfrak{D}(A)$ is again the infinitesimal generator of a semi-group of class $(1, \mathrm{~A})$ and

$$
T\left(\xi ; A_{1}\right)=\sum_{n=0}^{\infty} S_{n}(\xi), \quad \xi>0,
$$

where $S_{0}(\xi)=T(\xi ; A)$ and $S_{n}(\xi) x=\int_{0}^{\xi} T(\xi-\sigma ; A) \tilde{B} S_{n-1}(\sigma) x d \sigma ;$ the series converges absolutely, uniformly with respect to $\xi$ in each interval of the form $(\epsilon, 1 / \epsilon), 0<\epsilon<1$.

The series expansion (3.6) is essentially a power series expansion in $B$. The interpretation of the terms of this series is of some interest. The zero power term consists of the original semi-group operator at time $\xi$. The first power term consists of the original semi-group operator up to the time $\sigma$, an interaction with $B$ at this time, and this followed by the original semi-group operator acting on the soperturbed development for the remaining time $\xi-\sigma$; finally the resulting operators are averaged over all times $\sigma$ between 0 and $\xi$. The second power term consists of two interactions with $B$ and these averaged over all possible times of occurrence between 0 and $\xi$; etc. Theorem 3.1 can be proved by means of the corollary to Theorem 2.2.

As a consequence of the above result and our previous calculations, we can now state that the equation

$$
\begin{aligned}
\partial u / \partial \xi & =\partial^{2} u / \partial x^{2}+b(x) \partial u / \partial x, & -\infty<x<\infty, \xi>0 ; \\
\lim _{\xi \rightarrow 0+} u(\xi ; x) & =y_{0}(x), & y_{0} \in \mathfrak{D}(U),
\end{aligned}
$$

has a semi-group solution of class $(1, A)$ (actually of class $\left(\mathrm{C}_{0}\right)$ as we shall see below); here $b \in C[-\infty, \infty]$.

We shall say that $A_{1}$ is closely related to $A_{0}$ and write $A_{1} \sim A_{0}$, if $\mathfrak{D}\left(A_{1}\right)=\mathfrak{D}\left(A_{0}\right)$ and $B \equiv A_{1}-A_{0} \in \mathfrak{P}\left(A_{0}\right)$. It can be shown that this is an equivalence relation. Consequently if $\left[T\left(\xi ; A_{1}\right)\right]$ can be obtained from $\left[T\left(\xi ; A_{0}\right)\right]$ by a perturbation of the above kind, then $\left[T\left(\xi ; A_{0}\right)\right]$ can likewise be obtained from $\left[T\left(\xi ; A_{1}\right)\right]$ by such a perturbation. Moreover, any semi-group attained by a finite sequence of such perturbations can be obtained by a single perturbation of this kind.

Under this equivalence relation the set of all infinitesimal gener- 
ators of semi-groups of class $(1, A)$ splits up into equivalence classes $\left[\mathfrak{G}_{\alpha}\right]$. The following stable properties are shared by all members of an equivalence class if they belong to any member of this class.

(1) $A$ is a bounded linear operator;

(2) $A$ generates a semi-group of class $\left(\mathrm{C}_{0}\right)$;

(3) $A$ generates a group of linear bounded operators on $(-\infty, \infty)$;

(4) $T(\xi ; A)$ is continuous in the uniform operator topology for $\xi>0$.

Within each equivalence class it is possible to introduce a metric in such a way that the class becomes a complete metric space. To this end we choose a fixed $A_{\alpha} \in \mathcal{S}_{\alpha}$ and set

$$
d_{\alpha}\left(A_{1}, A_{2}\right) \equiv \int_{0}^{1}\left\|\left(A_{1}-A_{2}\right) T\left(\xi ; A_{\alpha}\right)\right\|_{A_{\alpha}} d \xi
$$

for all pairs $A_{1}, A_{2} \in \mathcal{S}_{\alpha}$. It can be shown that this function is a suitable metric function and that the resulting topology in $\mathbb{S}_{\alpha}$ is actually independent of the particular choice of $A_{\alpha}$ in $\mathfrak{S}_{\alpha}$. One can also define an equivalent topology for $\mathcal{E}_{\alpha}$ in terms of $R\left(\lambda ; A_{\alpha}\right)$ rather than $\left[T\left(\xi ; A_{\alpha}\right)\right]$ by means of the metric function

$$
\Delta_{\alpha}\left(A_{1}, A_{2}\right) \equiv \sup _{n \geqq 1} \int_{0}^{\infty} \frac{\lambda^{n-1}}{(n-1) !}\left\|\left(A_{1}-A_{2}\right) R^{(n)}\left(\lambda+\omega ; A_{\alpha}\right)\right\| d \lambda
$$

where $\omega$ is any fixed number greater than the type of $\left[T\left(\xi ; A_{\alpha}\right)\right]$.

We can now state the sense in which the generation problem is stable.

THEOREM 3.2. For the infinitesimal generators of a single equivalence class $\mathfrak{E}_{\alpha}$, we have

$$
\lim _{A \rightarrow A_{0}}\left\|T(\xi ; A)-T\left(\xi ; A_{0}\right)\right\|=0
$$

uniformly with respect to $\xi$ in each interval of the form $(\epsilon, 1 / \epsilon), 0<\epsilon<1$.

4. Semi-groups of class (A). It is of course desirable to obtain criteria for generating semi-groups more general than those of class $(1, A)$. The most far reaching result in this direction is due to $W$. Feller [3], who has found necessary and sufficient conditions that an operator generate a strongly continuous semi-group of linear bounded operators with range set $\mathfrak{X}_{0}$ dense in $\mathfrak{X}$; no assumption is made concerning the behavior of the semi-group in the neighborhood of $\xi=0$. For such semi-groups the operator which corresponds to our infinitesimal generator may have an empty resolvent set. For this and other reasons Feller's criteria can be verified only with difficulty. As a compromise between such extreme generality and semi-groups of 
class $(1, \mathrm{~A})$ we have proposed the following class.

Definition 4.1. A semi-group $\subseteq \equiv[T(\xi) ; \xi>0]$ of linear bounded operators will be said to be of class (A) if

(1) $T(\xi)$ is continuous in the strong operator topology for $\xi>0$;

(2) $\mathfrak{X}_{0}$ is dense in $\mathfrak{X}$;

(3) there exists a constant $\omega_{1}>\omega_{0}$ and a family of linear bounded operators $\left[R(\lambda) ; \Re(\lambda)>\omega_{1}\right]$ such that

(a) $\|R(\lambda)\|$ is bounded in the half-plane $\Re(\lambda)>\omega_{1}$,

(b) $\|R(\lambda)\|=O(1 / \lambda)$ as $\lambda \rightarrow \infty$,

(c) for each $x \in \mathfrak{X}_{0}$

$$
R(\lambda) x=\int_{0}^{\infty} e^{-\lambda \xi} T(\xi) x d \xi, \quad \Re(\lambda)>\omega_{1} .
$$

It follows from the properties already ascribed to semi-groups of class $(1, A)$ that such semi-groups are also of class (A). On the other hand there exist semi-groups of class (A) which are not of class $(1, \mathrm{~A}) .^{4}$

Semi-groups of class (A) satisfy a generalized initial value condition. In fact it can be shown that

$$
\lim _{\lambda \rightarrow \infty} \lambda R(\lambda) x=x, \quad x \in \mathfrak{X} .
$$

Thus $\mathfrak{S}$ is strongly Abel summable to the identity at $\xi=0$ in this extended sense. The infinitesimal operator $A_{0}$ again has a least closed extension $A$ and it turns out that $R(\lambda)=R(\lambda ; A)$ for all $\lambda$ with $\mathfrak{R}(\lambda)>\omega_{1}$; further $\mathfrak{D}(A) \supset \mathfrak{D}\left(A_{0}\right) \supset \mathfrak{D}\left(A^{3}\right)$. If $x \in \mathfrak{D}(A)$, then

$$
d T(\xi) x / d \xi=A_{0} T(\xi) x=T(\xi) A x,
$$

$\xi>0$

and if $x \in \mathfrak{D}\left(A^{2}\right)$, then $\lim _{\xi \rightarrow 0+} T(\xi) x=x$. The relations (4.2) and (4.3)

4 The following semi-group is of class (A) but not of class (1, A). Let $x$ consist of all sequence pairs $x=\left\{\left(\chi_{n}, \eta_{n}\right) ; n=1,2,3, \cdots\right\}$ such that $\lim _{n \rightarrow \infty} \chi_{n}=0$ and $\sum_{n=1}^{\infty} n^{2}\left|\eta_{n}\right|^{2}<\infty$ with norm $\|x\|=\sup _{n}\left|x_{n}\right|+\left[\sum_{n=1}^{\infty} n^{2}\left|\eta_{n}\right|^{2}\right]^{1 / 2}$. The operator $T(\xi) x=x^{\prime} \equiv\left\{\left(\chi_{n}^{\prime}, \eta_{n}^{\prime}\right)\right\}$ is defined by $\chi_{n}^{\prime}=\exp \left[-\left(n+i e^{n}\right) \xi\right]\left(\chi_{n} \cos n \xi-\eta_{n} \sin n \xi\right)$, $\eta_{n}^{\prime}=\exp \left[-\left(n+i e^{n}\right) \xi\right]\left(\chi_{n} \sin n \xi+\eta_{n} \cos n \xi\right)$. It is easy to show that $[T(\xi)]$ is a semigroup of linear bounded operators continuous in the strong operator topology for $\xi>0$. However, for $x=\{(1 / n, 0)\}$ we have $\|T(\xi) x\| \geqq c / \xi$ for $0<\xi<\pi / 2$ and hence $[T(\xi)]$ is not of class $(1, A)$. On the other hand, the ultimately zero vectors belong to $\mathfrak{X}_{0}$ and since these are dense in $\mathfrak{X}$ the same is true of $\mathfrak{X}_{0}$. Further, for $x \in \mathfrak{X}_{0}$, the operator $R(\lambda) x=x^{\prime}=\left\{\left(\chi_{n}^{\prime}, \eta_{n}^{\prime}\right)\right\}$ is given by $\chi_{n}^{\prime}=\alpha_{n}(\lambda) \chi_{n}-\beta_{n}(\lambda) \eta_{n}, \eta_{n}^{\prime}=\beta_{n}(\lambda) \chi_{n}+\alpha_{n}(\lambda) \eta_{n}$, where $\alpha_{n}(\lambda)=\left[\lambda+n+i e^{n}\right]\left[\left(\lambda+n+i e^{n}\right)^{2}+n^{2}\right]^{-1}, \beta_{n}(\lambda)=n\left[\left(\lambda+n+i e^{n}\right)^{2}+n^{2}\right]^{-1}$. From this one shows that $\left|\alpha_{n}(\sigma+i \tau)\right|,\left|\beta_{n}(\sigma+i \tau)\right| \leqq 1 / \sigma$ and $\left|\beta_{n}(\sigma+i \tau)\right|^{2} \leqq n^{2}\left[\sigma^{2}\left(\sigma^{2}\right.\right.$ $\left.\left.+\left(\tau+e^{n}\right)^{2}\right)\right]^{-1}$ for $\sigma>0$. These estimates can now be used to obtain (cf. R. S. Phillips $\left[13\right.$, p. 349]) $\sum_{n-1}^{\infty} n^{2}\left|\beta_{n}(\sigma+i \tau)\right|^{2} \leqq c_{1} \sigma^{-2}+c_{2} \sigma^{-3}$. Finally a straightforward calculation shows that $\|R(\sigma+i \tau)\| \leqq M / \sigma$ for $\sigma>1$. This proves that $[T(\xi)]$ is of class (A). 
show that $T(\xi) y_{0}$ for $y_{0} \in \mathfrak{D}(A)$ satisfies an abstract Cauchy problem. This problem is somewhat more general than that designated earlier as ACP inasmuch as $T(\xi) A y_{0} \equiv y^{\prime}\left(\xi ; y_{0}\right)$ need not be continuous for $\xi \geqq 0$, in fact it need not even be integrable on $(0,1)$.

For semi-groups of class (A) we have the following generation theorem.

TheOREM 4.1. A necessary and sufficient condition that a closed linear operator $U$ be the infinitesimal generator of a semi-group of class (A) with $\omega_{0}<\omega_{1}$ is that

(1) $\mathfrak{D}(U)$ is dense in $\mathfrak{X}$;

(2) $\|R(\lambda ; U)\|=O(1 / \lambda)$ as $\lambda \rightarrow \infty$;

(3) $R(\lambda ; U) \|$ is bounded for $\Re(\lambda)>\omega_{1}$;

(4) there exists a non-negative nonincreasing function $\phi(\xi) e^{-\omega 1 \xi}$ with $\lim \sup _{\xi \rightarrow \infty} \xi^{-1} \log \phi(\xi)<\omega_{1}$ which satisfies either of the following:

(a) for each $x \in \mathfrak{D}\left(U^{2}\right)$ there is a non-negative measurable function $\phi(\xi ; x) \leqq \phi(\xi)\|x\|$ such that $\int_{0}^{\infty} e^{-\omega_{1} \xi} \phi(\xi ; x) d \xi<\infty$ and $\left\|R^{(n)}(\lambda ; U) x\right\|$ $\leqq \int_{0}^{\infty} e^{-\lambda \xi} \xi^{n} \phi(\xi ; x) d \xi$ for all real $\lambda>\omega_{1}$ and integers $n \geqq 0$;

(b) let $x \in \mathfrak{D}\left(U^{2}\right)$ and $0<\delta^{\prime}<\delta$; for each $\epsilon>0$ there exists a $\lambda_{0}$ $\equiv \lambda_{0}\left(x ; \epsilon, \delta^{\prime}, \delta\right)$ such that if $\lambda>\lambda_{0}$ and $n \geqq \lambda \delta$, then

$$
\left\|\left(\lambda-\omega_{1}\right)^{n}[R(\lambda ; U)]^{n} x\right\| \leqq\left\{e^{-\omega_{1} \xi} \phi\left(\delta^{\prime}\right)+\epsilon\right\}\|x\| .
$$

Moreover $\|T(\xi)\| \leqq \phi(\xi)$ for all $\xi>0$.

Condition (4-b) of the above theorem was first used in connection with generation theorems by W. Feller [3]. Actually both (4-a) and (4-b) are difficult to verify in practice. The next theorem gives a much more workable criterion.

TheOREM 4.2. Let $\psi(\tau)$ be a positive-valued function defined and continuous on $(-\infty, \infty)$ such that

(a) $\psi(\tau) \rightarrow \infty$ as $|\tau| \rightarrow \infty$,

(b) $\psi^{\prime}(\tau)$ is bounded,

(c) $\int_{-\infty}^{\infty} \exp [-\xi \psi(\tau)] d \tau<\infty$ for each $\xi>0$.

Let $\omega$ be a given real constant. If $U$ is a closed linear operator with dense domain such that $R(\lambda ; U)$ exists for $\Re(\lambda) \geqq \omega$ and satisfies

(1) $\|R(\lambda ; U)\|$ is bounded for $\Re(\lambda) \geqq \omega$,

(2) $R(\lambda ; U) \|=O(1 / \lambda)$ as $\lambda \rightarrow \infty$,

(3) $\|R(\omega+i \tau ; U)\| \leqq M / \psi(\tau)$,

then $U$ generates a semi-group $[T(\xi)]$ of class (A) which is differentiable in the uniform operator topology for $\xi>0$.

The proof of Theorem 4.2 makes use of contour integration methods developed by E. Hille [4, Theorem 12.5.1]. 
As a special case of the above theorem we obtain the following criterion. Let $U$ be a closed linear operator with dense domain. If there exist real constants $\omega$ and $M$ such that

$$
\|R(\sigma+i \tau ; U)\| \leqq M\left(\sigma-\omega+|\tau|^{\beta}\right)^{-1}, \quad \sigma>\omega,
$$

where $\beta>0$, then $U$ generates a semi-group of class (A).

5. Adjoint theory. The collection $\mathfrak{X}^{*} \equiv\left[x^{*}\right]$ of complex-valued linear bounded functionals on $\mathfrak{X}$ forms a Banach space in which the vector operations are defined by $\left(\alpha x^{*}+\beta y^{*}\right)(x)=\alpha x^{*}(x)+\beta y^{*}(x)$ and the norm is given by $\left\|x^{*}\right\|=\sup \left[\left|x^{*}(x)\right| ;\|x\| \leqq 1\right]$. Likewise the linear bounded functionals on $\mathfrak{X}^{*}$ form a second adjoint space $\mathfrak{X}^{* *}$ and in this way a hierarchy of adjoint spaces can be constructed from the given Banach space $\mathfrak{X}$.

If $U$ is a closed linear operator on $\mathfrak{X}$ to itself with dense domain, an adjoint operator $U^{*}$ can be defined as follows: The domain $\mathfrak{D}\left(U^{*}\right)$ consists of the set of all $x^{*} \in \mathfrak{X}^{*}$ for which there exists a $y^{*} \in \mathfrak{X}^{*}$ such that $x^{*}[U x]=y^{*}(x)$ for all $x \in \mathfrak{D}(U)$; we set $U^{*} x^{*}=y^{*}$. It can be shown that $U^{*}$ is again a closed linear operator. If $U$ is bounded with $\mathfrak{D}(U)=\mathfrak{X}$, then $U^{*}$ is also bounded with $\mathfrak{D}\left(U^{*}\right)=\mathfrak{X}^{*}$; in this case a second adjoint operator $U^{* *}$ is definable as above and this process can be repeated indefinitely. However, if $U$ is merely closed with $\mathfrak{D}(U)$ dense in $\mathfrak{X}, \mathfrak{D}\left(U^{*}\right)$ need not be dense in $\mathfrak{X}^{*}$ and because of this fact the hierarchy of adjoint operators corresponding to $U$ will in general extend no further than $U^{*}$. By way of avoiding this situation, one may limit the adjoint space to $\left[\mathfrak{D}\left(U^{*}\right)\right]^{-}$, which we denote by $\mathfrak{X}^{\odot}$, and take $U^{\odot}$ to be the restriction of $U^{*}$ having domain $\mathfrak{D}\left(U^{\odot}\right)=\left[x^{*} ; x^{*} \in \mathfrak{D}\left(U^{*}\right), U^{*} x^{*} \in \mathfrak{X}^{\odot}\right]$. Then, supposing $\mathfrak{D}\left(U^{\odot}\right)$ to be. dense in $\mathfrak{X}^{\odot}$, one may proceed to define a second "adjoint" operator $U^{\odot \odot}$ on the subspace $\mathfrak{X}^{\odot} \odot \equiv \mathfrak{D}\left[\left(U^{\odot}\right)^{*}\right]-$ of $\left(\mathfrak{X}^{\odot}\right)^{*}$. Now if $U$ is such that $\mathfrak{D}(U)$ is dense in $\mathfrak{X}$ and $\|R(\lambda ; U)\|=O(1 / \lambda)$ as $\lambda \rightarrow \infty$ then it can be shown that this process can be continued indefinitely and one obtains an adjoint theory relative to $U$ which is an analogue of the usual adjoint theory.

There are two rather natural ways in which this adjoint theory can

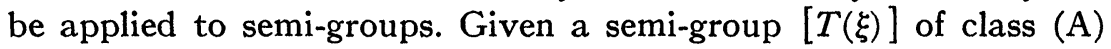
and with infinitesimal generator $A$, we can either take the adjoint semi-group to be the semi-group generated by $A^{\odot}$ having as its domain $\mathfrak{X}^{\odot}=\left[\mathfrak{D}\left(A^{*}\right)\right]$-, or we can take it to be the semi-group of adjoint operators $\left[T^{*}(\xi)\right]$ with domain $\mathfrak{X}^{*}$. When $\mathfrak{X}^{\odot}$ is a proper subspace of $\mathfrak{X}^{*}$ these two notions are distinct. We have chosen to treat the infinitesimal generator as the primary entity, and from this point of view the proper domain of the adjoint semi-group is $\mathfrak{X}^{\odot}$. 
It turns out that the semi-group generated by $A^{\odot}$ is again of class (A) and that the member operators are just the restrictions of the adjoint operators $T^{*}(\xi)$ to the domain $\mathfrak{X}^{\odot}$. As to the other alternative, while it is true that the adjoint operators $\left[T^{*}(\xi)\right]$ with domain $\mathfrak{X}^{*}$ form a semi-group, this semi-group will in general not be continuous in the strong operator topology and hence our basic theory would not apply. Actually $\mathfrak{X}^{\odot}$ is the largest closed subspace of $\mathfrak{X}^{*}$ on which $\left[T^{*}(\xi)\right]$ is strongly continuous and in which the corresponding range space is dense.

The importance of the adjoint semi-group appears to have been first recognized by W. Feller [1] in his treatise on the parabolic differential equation. In the special problem with which he was dealing, he was able to obtain the full adjoint semi-group without employing a precise notion for the adjoint to an unbounded operator. The general treatment sketched above is due to R. S. Phillips [15].

6. Operational calculus and spectral theory. The extension of the operational calculus to closed linear operators with nonempty resolvent sets goes back to A. E. Taylor [17], who discovered the basic integral representation for the associated mapping. This calculus can be described as follows:

Let $\Delta$ be an open subset of the extended complex plane not containing the point $\alpha_{0}, \alpha_{0} \neq \infty$. Let $\mathfrak{S}(\Delta)$ be the complex algebra of all functions $f(\lambda)$ locally holomorphic in $\Delta$ with the sequence topology: $f_{n} \rightarrow f$ denoting that $f_{n}(\lambda)$ converges pointwise to $f(\lambda)$, uniformly in each compact subset of $\Delta$. Further let $\mathfrak{B}(\Delta)$ be the algebra of functions $f(A)$ with domain $\mathfrak{B}(\Delta) \equiv\left[A ; \sigma_{e}(A) \subset \Delta\right]$ and range $\mathbb{E}(\mathfrak{X}) .{ }^{5}$ Then there exists an isomorphic mapping: $f(\lambda) \rightarrow f(A)$ of $\mathfrak{S}(\Delta)$ into $\mathfrak{B}(\Delta)$ such that (i) $1 \rightarrow I$, (ii) $\left(\alpha_{0}-\lambda\right)^{-1} \rightarrow R\left(\alpha_{0} ; A\right)$, and (iii) $f_{n} \rightarrow f$ implies that $\left\|f_{n}(A)-f(A)\right\|$ $\rightarrow 0$ for each $A \in \mathbb{S}(\Delta)$. This mapping is unique and is represented by

$$
f(A)=\delta f(\infty) I+\frac{1}{2 \pi i} \int_{\mathbf{r}_{\mathbf{A}}} f(\zeta) R(\zeta ; A) d \zeta
$$

where $\Gamma_{A}$ is an oriented envelope of $\sigma_{e}(A)$ contained in $\Delta$ and $\delta=1$ or 0 according as $\Gamma_{A}$ contains $\zeta=\infty$ in its interior or not.

By suitably defining a notion of analyticity, it is possible to restrict $\mathfrak{B}(\Delta)$ in the above theorem to functions $f(A)$ which are locally analytic on $\mathbb{S}(\Delta)$ to $\mathbb{E}(\mathfrak{X})$.

If we limit our consideration to operators which are infinitesimal

5 We denote the spectrum of $A$ by $\sigma(A)$ and the extended spectrum of $A$ by $\sigma_{\theta}(A)$. If $A$ is bounded $\sigma_{\theta}(A)=\sigma(A)$ whereas if $A$ is unbounded $\sigma_{\theta}(A)=\sigma(A) \bigcup_{\infty}$. ( $(\mathfrak{X}(\mathfrak{X})$ denotes the algebra of all linear bounded operators on $X$ to itself. 
generators of semi-groups of class (A), we can devise an operational calculus for this restricted class of operators which, so to speak, permits $\sigma_{e}(A)$ to have a point on the boundary of $\Delta$. Actually the defining entity, instead of being the domain $\Delta$, is now a submultiplicative function $^{6} \phi(\xi)$ of type

$$
\omega_{0} \equiv \lim _{\xi \rightarrow \infty} \xi^{-1} \log \phi(\xi)>-\infty .
$$

Each function $f(\lambda)$ is required to be the Laplace-Stieltjes transform of a set function belonging to the algebra $S(\phi)$ which is defined as follows:

DEFINITION 6.1. Let $S(\phi)$ be the family of countably additive complex-valued set functions on the Borel subsets of $[0, \infty)$ such that $\int_{0}^{\infty} \phi(\xi)|d a|<\infty$. For such a set function

$$
\psi(a ; \lambda) \equiv \int_{0}^{\infty} e^{\lambda \xi} d a
$$

exists for all $\lambda$ with $\Re(\lambda) \leqq \omega_{0}$. Let $Q\left(\omega_{0}\right)$ consist of the elements in $S\left(e^{\omega_{0} \xi}\right)$ such that $\psi\left(a ; \omega_{0}+i \tau\right) \in L(-\infty, \infty)$ as a function of $\tau$; and let $\alpha_{0}$ be a fixed complex number with $\Re\left(\alpha_{0}\right)>\omega_{0}$. Then

$$
S(\phi) \equiv S(\phi)+Q\left(\omega_{0}\right)+S(\phi) * r_{\alpha_{0}}
$$

where $r_{\alpha}(E)=\int_{E} e^{-\alpha \xi} d \xi$ and $*$ denotes convolution.

On introducing a product in $S(\phi)$ defined by convolution, $S(\phi)$ becomes a subalgebra of $S\left(e^{\omega_{0} \xi}\right)$. Further $S(\phi)$ is independent of our particular choice of $\alpha_{0}$. We note that $S\left(e^{\omega_{0} \xi}\right)$ is the adjoint space to the space of continuous functions $f(\xi), \xi \geqq 0$, with $\lim _{\xi \rightarrow \infty} e^{-\omega_{0} \xi} f(\xi)=0$; the norm being given by $\|f\|=\sup _{\xi \geq 0}\left|e^{-\omega_{0} \xi} f(\xi)\right|$. Consequently $S\left(e^{\omega_{0} \xi}\right)$ has a well defined weak* topology.

The domain of the operational calculus is limited by $\phi(\xi)$ in the following manner.

Definition 6.2. Let $[T(\xi ; A)]$ be a semi-group of class (A) and type

and set

$$
\omega_{0}(A) \equiv \lim _{\xi \rightarrow \infty} \xi^{-1} \log \|T(\xi ; A)\|,
$$

Then if $\|T(\xi ; A)\| \leqq \phi(\xi)$ and if $\omega_{1}(A)<\omega_{0}$ we say that $A$ is strictly majorized by $\phi(\xi)$ and we denote this relation by $A<\phi$. Finally we set $\mathfrak{A}_{0}(\phi) \equiv[A ; A<\phi]$.

- We suppose that $\phi(\xi)$ is a real-valued Borel measurable function defined on $[0, \infty)$ such that $0<\phi\left(\xi_{1}+\xi_{2}\right) \leqq \phi\left(\xi_{1}\right) \phi\left(\xi_{2}\right)$ for all $\xi_{1}, \xi_{2}>0$, with $\phi(0)=1$. 
The manner in which the elements of $S(\phi)$ operate on $A$ is given by

Definition 6.3. Let $\mathfrak{X}_{1}(A) \equiv\left[x ; \lim _{\xi \rightarrow 0+} T(\xi ; A) x=x\right]$. For $a \in \mathcal{S}(\phi)$, $A \in \mathfrak{P}_{0}(\phi)$, and $x \in \mathfrak{X}_{1}(A)$ we define

$$
\Psi(a ; A) x=\int_{0}^{\infty} T(\xi ; A) x d a
$$

or, as an alternative,

$$
\Psi(a ; A) x=\frac{1}{2 \pi i}(C, 1)-\int_{\gamma-i \infty}^{\gamma+i \infty} \psi(a ; \zeta) R(\zeta ; A) x d \zeta+\frac{1}{2} a([0]) x
$$

where $\omega_{1}(A)<\gamma \leqq \omega_{0}$.

It can be shown that the relations (6.7) and (6.8) define the same linear bounded operator on $\mathfrak{X}$. We note that in general $\psi(a ; \zeta)$ is singular at infinity and that infinity belongs to $\sigma_{e}(A)$; this can not occur in the calculus associated with the relation (6.1).

We can now state our principal result.

THEOREM 6.1. Let $\phi(\xi)$ be a submultiplicative function of type $\omega_{0}$ $>-\infty$ and suppose $\alpha_{0}$ is such that $\Re\left(\alpha_{0}\right)>\omega_{0}$. Let $S(\phi)$ be the algebra of set functions described in Definition 6.1 with a sequence topology: $a_{n} \rightarrow a$ denoting that the sequence $\left\{a_{n}\right\}$ converges to $a$ in the weak* topology of $S\left(e^{\omega_{0} \xi}\right)$. Further let $\mathfrak{B}(\phi)$ be the complex algebra of functions $f(A)$ defined on $\mathfrak{A}_{0}(\phi)$ and having values in $\mathfrak{E}(\mathfrak{X})$, the arithmetic operations being defined as in $\mathbb{E}(\mathfrak{X})$.

There exists an isomorphic mapping: $a \rightarrow \Psi(a ; A)$ of $\mathcal{S}(\phi)$ onto a subalgebra of $\mathfrak{B}(\phi)$ such that (i) the unit $e_{0} \in \mathcal{S}(\phi) \rightarrow I$; (ii) $r_{\alpha_{0}} \rightarrow R\left(\alpha_{0} ; A\right)$; and (iii) $a_{n} \rightarrow a$ implies that $\Psi\left(a_{n} ; A\right) x \rightarrow \Psi(a ; A) x$ for each $x \in \mathfrak{X}_{1}(A)$. This mapping is unique and is defined by (6.7) or, alternately, by (6.8) for each $x \in \mathfrak{X}_{1}(A)$.

By limiting the above assertion to the set $\mathfrak{A}_{1}$ of all infinitesimal generators of semi-groups of class $(1, A)$, rather than of class (A), a notion of analyticity can be introduced so as to make the functions $\Psi(a ; A) \subset \mathfrak{B}(\phi)$ locally analytic in $\mathfrak{A}_{1}(\phi) \equiv \operatorname{Int}\left[\mathfrak{H}_{0}(\phi) \cap \mathfrak{A}_{1}\right]$.

The extension of the operational calculus to unbounded operatorvalued functions appears to be a very promising field for further research. The first results in this direction are to be found in a paper by R. S. Phillips [11]; a more systematic treatment has been given by A. V. Balakrishnan (University of Southern California thesis, 1954). A somewhat different approach to the subject employing the theory of distributions has also been developed by L. Schwartz (unpublished). 
Having developed an operational calculus for semi-groups of class (A), we now consider the relation between the spectrum of $\Psi(a ; A)$ and that of $A$, where $a \in \mathcal{S}(\phi)$ and $A<\phi$. Our results, although more general, follow the same pattern as those previously obtained by E. Hille [4] and R. S. Phillips [10] both as to content and method of proof.

It can be shown for $a \in \mathcal{S}(\phi)$ and $A<\phi$ that

$$
[\psi(a ; \sigma(A))]-\subset \sigma[\Psi(a ; A)],
$$

where the inclusion can be proper. If the set function or the infinitesimal generator is sufficiently specialized, a more precise spectral mapping theorem can be proved. For instance, if $a$ is an absolutely continuous element of $S(\phi)$ and $A<\phi$ is otherwise arbitrary, then $\psi(a ; \sigma(A)) \cup 0=\sigma[\Psi(a ; A)]$. Likewise if $a \in \mathcal{S}(\phi)$, but is otherwise not restricted, and if $A \prec \phi$ is the infinitesimal generator of a semigroup continuous in the uniform operator topology for $\xi \geqq \gamma>0$, then $\psi(a ; \sigma(A)) \cup a(\{0\})=\sigma[\Psi(a ; A)]$. In both of these cases even the fine structure of the spectrum is preserved under the mapping.

Of special interest is the relation between the spectrum of $T(\xi ; A)$ and that of $A$. In view of the above results one would expect that $\sigma[T(\xi ; A)]$ would essentially coincide with $\exp [\xi \sigma(A)]$ with possibly a $\lambda=0$ in $\sigma[T(\xi ; A)]$ being associated with a $\lambda=\infty$ in $\sigma_{e}(A)$. Actually, aside from the latter possibility, the correspondence for the point and residual spectra is very good. However, the continuous spectrum of $T(\xi ; A)$ comes in part from the continuous spectrum of $A$ and in part from the limit points of the set $\exp [\xi \sigma(A)]$ which are not otherwise accounted for. The continuous spectrum of $T(\xi ; A)$ may also contain points which have no relation whatever with the points of $\sigma_{e}(A)$. For example, there exists a strongly continuous group of linear bounded operators $[T(\xi ; A) ;-\infty<\xi<\infty]$ such that $\sigma(A)$ is empty, $\sigma_{\theta}(A)$ containing only the point at infinity; on the other hand $\sigma[T(\xi ; A)]$ which is obviously nonempty does not contain the only point, namely $\lambda=0$, that could reasonably correspond to $\sigma_{e}(A)$.

\section{REFERENCES}

1. W. Feller, The parabolic differential equations and the associated semi-groups of transformations, Ann. of Math. vol. 55 (1952) pp. 468-519.

2. - Semi-groups of transformations in general weak topologies, Ann. of Math. vol. 57 (1953) pp. 287-308.

3. - On the generation of unbounded semi-groups of bounded linear operators, Ann. of Math. vol. 58 (1953) pp. 166-174.

4. E. Hille, Functional analysis and semi-groups, Amer. Math. Soc. Colloquium Publications, vol. 31, New York, 1948. 
5. - On the generation of semi-groups and the theory of conjugate functions, Kungl. Fysiografiska Sällskapets I Lund Förhandlingar vol. 21 (14) (1952) pp. 1-13. 6. - Le problème abstrait de Cauchy, Università di Torino, Rendiconti del Seminario Mat. vol. 12 (1953) pp. 95-103.

7. - The abstract Cauchy problem and Cauchy's problem for parabolic differential equations, Journal d'Analyse Mathématiques vol. 3 (1954) pp. 83-196.

8. I. Miyadera, Generation of a strongly continuous semi-group of operators, Tôhoku Math. J. vol. 4 (2) (1952) pp. 109-114.

9. R. S. Phillips, On one parameter semi-groups of linear transformations, Proc. Amer. Math. Soc. vol. 2 (1951) pp. 234-237.

10. - Spectral theory for semi-groups of linear operators, Trans. Amer. Math. Soc. vol. 71 (1951) pp. 393-415.

11. - On the generation of semi-groups of linear operators, Pacific Journal of Mathematics vol. 2 (1952) pp. 343-369.

12. - Perturbation theory for semi-groups of linear operators, Trans. Amer. Math. Soc. vol. 74 (1953) pp. 199-221.

13. - An inversion formula for Laplace transforms and semi-groups of linear operators, Ann. of Math. vol. 59 (1954) pp. 325-356.

14. - A note on the abstract Cauchy problem, Proc. Nat. Acad. Sci. U.S.A. vol. 40 (1954) pp. 244-248.

15. - The adjoint semi-group, to appear in the Pacific Journal of Mathematics.

16. M. H. Stone, Linear transformations in Hilbert space III, Proc. Nat. Acad. Sci. U.S.A. vol. 16 (1930) pp. 172-175.

17. A. E. Taylor, Spectral theory of closed distributive operators, Acta Math. vol. 84 (1950) pp. 189-224.

18. D. V. Widder, The Laplace transform, Princeton University Press, 1941.

19. $\mathrm{K}$. Yosida, On the differentiability and the representation of one-parameter semigroups of linear operators, J. Math. Soc. of Japan vol. 1 (1948) pp. 15-21.

20. - Integration of Fokker-Planck's equation with a boundary condition, J. Math. Soc. of Japan vol. 3 (1951) pp. 69-73.

21. - On the integration of diffusion equations in Riemann spaces, Proc. Amer. Math. Soc. vol. 3 (1952) pp. 864-873.

The University of Southern California and STANFORD UNIVERSITY 